\title{
Holomorphic functions in generalized Cayley-Dickson algebras
}

\author{
Cristina Flaut and Vitalii Shpakivskyi
}

\begin{abstract}
In this paper we investigated some properties of holomorphic functions (belonging to the kernel of the Dirac operator) defined on domains of the real Cayley-Dickson algebras. For this purpose, we study first some properties of these algebras, especially multiplication tables for certain elements of the basis. Using these properties, we provided an algorithm for constructing examples of the class of functions under consideration.
\end{abstract}

Keywords: Cayley-Dickson and generalized Cayley-Dickson algebras; Dirac operator; holomorphic functions.

AMS Subject Classification: 30G35, 17A20.

\section{Introduction}

The theory of quaternionic differentiable functions has many applications in different areas of mathematics, physics and in other applied sciences (see, for example, [3], 4]). This theory has its origins in the paper [7] in which the authors proposed, for the first time, an analogue of the Cauchy-Riemann conditions in three-dimensional case. For the four-dimensional case, an analogue of these conditions was considered in the paper [2] and, as a next step of this generalization, the differentiable functions in the octonionic algebra was considered in the papers [11, [12.

Generalization of the Cauchy-Riemann conditions in all algebras obtained by the Cayley-Dickson process (called Cayley-Dickson algebras) was done in the paper [6], where differentiable functions of variables belonging to Cayley-Dickson algebras were defined. For such functions, was established analogues results with the main results of complex analysis, results which can be successfully used in the further studies of special functions of variables with values in CayleyDickson algebras.

Comparing with [6], in the present paper, we investigate another class of differentiable functions (using the Dirac operator) in Cayley-Dickson algebras and, more important, we provide an example of this kind of functions and an 
algorithm to find such as examples. Since these functions are rather complicated objects, it is quite important to have a way to generate examples.

The paper is organized in two sections. In the first section, we briefly presented some properties of algebras obtained by the Cayley-Dickson process and the algorithm described by J. W. Bales regarding to an easy way to multiply the elements from a basis in such algebras (by using exclusive or operation and a twist map). In the second section, by description the multiplication tables for certain elements of the basis (Propositions 2.2 and 2.3), we obtained the main result of this work: an example of a left hyperholomorphic function in generalized Cayley-Dickson algebras (Theorem 2.12). Moreover, in the Thorem 2.10 we proved that for studying left $A_{t}$-holomorphic functions in generalized CayleyDickson algebras $A_{t}=\left(\frac{\gamma_{1}, \ldots, \gamma_{t}}{\mathbb{R}}\right)$ it is suffices to consider left $A_{t}$-holomorphic functions only in the algebras $\left(\frac{\operatorname{sign}\left(\gamma_{1}\right), \ldots, \operatorname{sign}\left(\gamma_{t}\right)}{\mathbb{R}}\right)$, that means this study depends only by the sign of the real numbers $\gamma_{1}, \gamma_{2}, \ldots, \gamma_{t}$.

\section{Preliminaries}

Let $K$ be a commutative field with $\operatorname{char} K \neq 2$ and $A$ be an algebra over the field $K$. A unitary algebra $A \neq K$ such that we have $x^{2}+\alpha_{x} x+\beta_{x}=0$, for each $x \in A$, with $\alpha_{x}, \beta_{x} \in K$, is called a quadratic algebra.

In the following, we briefly present the Cayley-Dickson process and the properties of the algebras obtained. For details about the Cayley-Dickson process, the reader is referred to [9] and [10].

Let $A$ be a finite dimensional unitary algebra over a field $K$ with a scalar involution

$$
\text { - }: A \rightarrow A, \quad a \rightarrow \bar{a},
$$

i. e. a linear map satisfying the following relations:

$$
\overline{a b}=\bar{b} \bar{a}, \quad \overline{\bar{a}}=a,
$$

and

$$
a+\bar{a}, a \bar{a} \in K \cdot 1 \text { for all } a, b \in A .
$$

The element $\bar{a}$ is called the conjugate of the element $a$, the linear form

$$
t: A \rightarrow K, \quad t(a)=a+\bar{a}
$$

and the quadratic form

$$
n: A \rightarrow K, \quad n(a)=a \bar{a}
$$

are called the trace and the norm of the element $a$. Hence an algebra $A$ with a scalar involution is quadratic.

Let $\gamma \in K$ be a fixed non-zero element. We define the following algebra multiplication on the vector space

$$
A \oplus A:\left(a_{1}, a_{2}\right)\left(b_{1}, b_{2}\right):=\left(a_{1} b_{1}+\gamma b_{2} \overline{a_{2}}, \overline{a_{1}} b_{2}+b_{1} a_{2}\right) .
$$


We obtain an algebra structure over $A \oplus A$, denoted by $(A, \gamma)$ and called the algebra obtained from $A$ by the Cayley-Dickson process or simply generalized Cayley-Dickson algebra. We have $\operatorname{dim}(A, \gamma)=2 \operatorname{dim} A$.

Let $x \in(A, \gamma), x=\left(a_{1}, a_{2}\right)$. The map

$$
\text { - }:(A, \gamma) \rightarrow(A, \gamma), x \rightarrow \bar{x}=\left(\bar{a}_{1},-a_{2}\right),
$$

is a scalar involution of the algebra $(A, \gamma)$, extending the involution ${ }^{-}$of the algebra $A$.

If we take $A=K$ and apply this process $t$ times, $t \geq 1$, we obtain an algebra over $K$,

$$
A_{t}=\left(\frac{\gamma_{1}, \ldots, \gamma_{t}}{K}\right)
$$

By induction in this algebra, the set $\left\{e_{0}=1, e_{1}, \ldots, e_{n-1}\right\}, n=2^{t}$, generates a basis with the properties:

$$
e_{i}^{2}=\gamma_{i} 1, \gamma_{i} \in K, \gamma_{i} \neq 0, i=1, \ldots, n-1
$$

and

$$
e_{i} e_{j}=-e_{j} e_{i}=\beta_{i j} e_{k}, \beta_{i j} \in K, \beta_{i j} \neq 0, \quad i \neq j, \quad i, j=1, \ldots n-1,
$$

$\beta_{i j}$ and $e_{k}$ being uniquely determined by $e_{i}$ and $e_{j}$.

From [10, Lemma 4, it results that in an algebra $A_{t}$ with the basis $B=$ $\left\{e_{0}=1, e_{1}, \ldots, e_{n-1}\right\}$ satisfying relations (22) and (3) we have:

$$
e_{i}\left(e_{i} x\right)=\gamma_{i}^{2} x=\left(x e_{i}\right) e_{i},
$$

for all $i \in\{1,2, \ldots, n-1\}$ and for every $x \in A$.

The algebras $A_{t}$, in general, are neither commutative and nor associative algebras, but are flexible (i. e. $x(y x)=(x y) x=x y x$, for all $x, y \in A_{t}$ ) quadratic and power associative (i. e. the subalgebra $\langle x\rangle$ of $A$, generated by any element $x \in A$, is associative).

Remark 1.1. For $\gamma_{1}=\ldots=\gamma_{t}=-1$ and $K=\mathbb{R}$, in [1], the author described how we can multiply the basis vectors in the algebra $A_{t}, \operatorname{dim} A_{t}=$ $2^{t}=n$. He used the binary decomposition for the subscript indices.

Let $e_{p}, e_{q}$ be two vectors in the basis $B$ with $p, q$ representing the binary decomposition for the indices of the vectors, that means $p, q$ are in $\mathbb{Z}_{2}^{n}$. We have that $e_{p} e_{q}=\gamma_{n}(p, q) e_{p \otimes q}$, where:

i) $p \otimes q$ are the sum of $p$ and $q$ in the group $\mathbb{Z}_{2}^{n}$ or, more precisely, the "exclusive or" for the binary numbers $p$ and $q$;

ii) $\gamma_{n}$ is a function $\gamma_{n}: \mathbb{Z}_{2}^{n} \times \mathbb{Z}_{2}^{n} \rightarrow\{-1,1\}$.

The map $\gamma_{n}$ is called the twist map.

The elements of the group $\mathbb{Z}_{2}^{n}$ can be considered as integers from 0 to $2^{n}-1$ with multiplication "exclusive or" of the binary representations. Obviously, this operation is equivalent with the addition in $\mathbb{Z}_{2}^{n}$. 
From now on, in whole the paper, we will consider $K=\mathbb{R}$. Using the same notations as in the Bales's paper, we consider the following matrices:

$$
A_{0}=A=\left(\begin{array}{cc}
1 & 1 \\
1 & -1
\end{array}\right), \quad B=\left(\begin{array}{cc}
1 & -1 \\
1 & 1
\end{array}\right), \quad C=\left(\begin{array}{cc}
1 & -1 \\
-1 & -1
\end{array}\right) .
$$

In the same paper [1, the author find the properties of the twist map $\gamma_{n}$ and put the signs of this map in a table. He partitioned the twist table for $\mathbb{Z}_{2}^{n}$ into $2 \times 2$ matrices and obtained the following result:

Theorem 1.2. ([1], Theorem 2.2., p. 88-91) For $n>0$, the CayleyDickson twist table $\gamma_{n}$ can be partitioned in quadratic matrices of dimension 2 of the form $A, B, C,-B,-C$, defined in the relation (5). Relations between them can be found in the below twist trees:
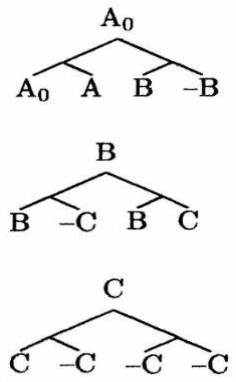
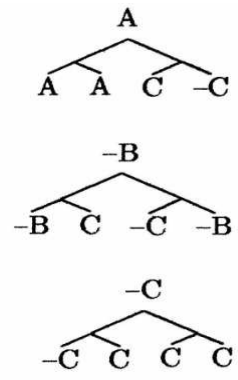

Fig. 1: Twist trees([1], Table 9)

Definition 1.3. Let $x=x_{0}, x_{1}, x_{2}, \ldots$ and $y=y_{0}, y_{1}, y_{2}, \ldots$. be two sequences of real numbers. The ordered pair

$$
(x, y)=x_{0}, y_{0}, x_{1}, y_{1}, x_{2}, y_{2}, \ldots
$$

is a sequence obtained by shuffling the sequences $x$ and $y$.

Using Theorem 1.2, in [1], the author gave the below algorithm for find $\gamma_{n}(s, r)$, where $s, r \in \mathbb{Z}_{2}^{n}$ :

i) We find the shuffling sequence $(s, r)$.

ii) Starting with the root $A_{0}$, we can find $\gamma_{n}(s, r)$ using the twist tree. We remark that "00" = unchanged, "01" = left $\rightarrow$ right, "10" =right $\rightarrow$ left, $" 11 "=$ right $\rightarrow$ right.

Let $\mathbb{H}\left(\gamma_{1}, \gamma_{2}\right)$ be the generalized quaternion algebra and $\mathbb{H}(-1,-1)$ be the quaternion division algebra. Below, you can see the multiplication tables: 


\begin{tabular}{l|ll|ll|}
$\cdot$ & 1 & \multicolumn{1}{l}{$e_{1}$} & $e_{2}$ & $e_{3}$ \\
\hline 1 & 1 & $e_{1}$ & $e_{2}$ & $e_{3}$ \\
$e_{1}$ & $e_{1}$ & $\gamma_{1}$ & $e_{3}$ & $\gamma_{1} e_{2}$ \\
\cline { 2 - 5 }$e_{2}$ & $e_{2}$ & $-e_{3}$ & $\gamma_{2}$ & $-\gamma_{2} e_{1}$ \\
$e_{3}$ & $e_{3}$ & $-\gamma_{1} e_{2}$ & $\gamma_{2} e_{1}$ & $\gamma_{1} \gamma_{2}$ \\
\cline { 2 - 5 }
\end{tabular}

Multiplication table for the generalized quaternion algebra

\begin{tabular}{l|ll|ll}
$\cdot$ & 1 & $e_{1}$ & $e_{2}$ & $e_{3}$ \\
\hline 1 & 1 & $e_{1}$ & $e_{2}$ & $e_{3}$ \\
$e_{1}$ & $e_{1}$ & -1 & $e_{3}$ & $-e_{2}$ \\
\cline { 2 - 5 }$e_{2}$ & $e_{2}$ & $-e_{3}$ & -1 & $e_{1}$ \\
$e_{3}$ & $e_{3}$ & $e_{2}$ & $-e_{1}$ & -1 \\
\cline { 2 - 5 } & & &
\end{tabular}

Multiplication table for the real division quaternion algebra

\begin{tabular}{l|ll|ll|}
$\cdot$ & 1 & $e_{1}$ & $e_{2}$ & $e_{3}$ \\
\hline 1 & 1 & 1 & 1 & 1 \\
$e_{1}$ & 1 & -1 & 1 & -1 \\
\cline { 2 - 5 }$e_{2}$ & 1 & -1 & -1 & 1 \\
$e_{3}$ & 1 & 1 & -1 & -1 \\
\cline { 2 - 4 } & &
\end{tabular}

Quaternion twist table

$\left(\begin{array}{cc}A_{0} & A \\ B & -B\end{array}\right)$

Quaternion twist table using notations from Theorem 1.2.

Example 1.4. Let $A_{4}$ be the real sedenion algebra. That means $\operatorname{dim} A_{4}=$ 16 with $\left\{1, e_{1}, \ldots, e_{15}\right\}$ a basis in this algebra. Let compute $e_{7} e_{13}=\gamma_{4}\left(7_{2}, 13_{2}\right) e_{7 \otimes 13}$. We have the following binary decompositions:

$$
\begin{aligned}
7_{2} & =0111, \text { since } 7=2^{2}+2+1 \text { and } \\
13_{2} & =1101, \text { since } 13=2^{3}+2^{2}+1 .
\end{aligned}
$$

Since $0111 \otimes 1101=1010\left(=2^{3}+2=10\right)$, it results that $7 \otimes 13=10$.

Now, we compute $\gamma_{4}\left(e_{7}, e_{13}\right)$. First, we shuffle the sequences 0111 and 1101. We obtain 01111011 . Starting with $A_{0}$, it results: $A_{0} \stackrel{01}{\rightarrow} A \stackrel{11}{\rightarrow}-C \stackrel{10}{\rightarrow} C \stackrel{11}{\rightarrow}-C$, then $\gamma_{4}\left(e_{7}, e_{13}\right)=-1$ and $e_{7} e_{13}=-e_{10}$. 


\section{Main results}

In this section, for a generalized Cayley-Dickson algebra $A_{t}$, writing the basis's elements in a convenient way, we can obtain multiplication tables for certain elements of the basis. Using these results, in Theorem 2.12 we provide an example of a left hyperholomorphic function in generalized Cayley-Dickson algebras.

Remark 2.1. i) In the generalized quaternion algebra, $\mathbb{H}\left(\gamma_{1}, \gamma_{2}\right)$, the basis can be written as

$$
\left\{1=e_{0}, e_{1}, e_{2}, e_{1} e_{2}\right\}
$$

For the generalized octonion algebra, $\mathbb{O}\left(\gamma_{1}, \gamma_{2}, \gamma_{3}\right)$, the basis can be written

$$
\left\{1=e_{0}, e_{1}, e_{2}, e_{1} e_{2}, e_{4}, e_{1} e_{4}, e_{2} e_{4},\left(e_{1} e_{2}\right) e_{4}\right\} .
$$

Therefore $e_{3}=e_{1} e_{2}, e_{7}=e_{3} e_{4}=\left(e_{1} e_{2}\right) e_{4}, e_{2} e_{4}=e_{6}$ and, when compute them, in these products do not appear any of the elements $\gamma_{1}, \gamma_{2}, \gamma_{3}$, or products of some of them at the end.

We remark that in the algebra $A_{t}=\left(\frac{\gamma_{1}, \ldots, \gamma_{t}}{\mathbb{R}}\right)$ in the products of the form

$$
e_{1} e_{2},\left(e_{1} e_{2}\right) e_{4}, \ldots,\left(\left(e_{2^{r}} e_{2^{r+1}}\right) \ldots e_{2^{k}}\right) e_{2^{i}}
$$

when compute them, do not appear any of the elements $\gamma_{1}, \gamma_{2}, \ldots, \gamma_{t}$ or products of some of them at the end.

ii) Using above remarks, the basis in the algebra $A_{t}=\left(\frac{\gamma_{1}, \ldots, \gamma_{t}}{\mathbb{R}}\right)$ can be written under the form

$$
\left\{1=e_{0}, e_{1}, e_{2}, \ldots, e_{2^{t-1}-1}, e_{2^{t-1}}, e_{1} e_{2^{t-1}}, e_{2} e_{2^{t-1}}, e_{3} e_{2^{t-1}}, \ldots, e_{2^{t-1}-1} e_{2^{t-1}}\right\}
$$

with

$$
e_{i} e_{2^{t-1}}=-e_{2^{t-1}} e_{i}=e_{2^{t-1}} \bar{e}_{i}, \quad i \in\left\{1,2, \ldots, 2^{t-1}-1\right\} .
$$

Proposition 2.2. Let $A_{t}=\left(\frac{\gamma_{1}, \ldots, \gamma_{t}}{\mathbb{R}}\right)$ be an algebra obtained by the CayleyDickson process and $\left\{e_{0}=1, e_{1}, \ldots, e_{n-1}\right\}, n=2^{t}$ be a basis. Let $r \geq 1, r<$ $k \leq i<t$. Therefore

$$
\begin{aligned}
\left(\left(e_{2^{r}} e_{2^{r+1}}\right) \ldots e_{2^{k}}\right) e_{2^{i}} & =(-1)^{k-r+2} e_{T}, \\
\left.\left(\left(e_{1} e_{2^{r}}\right) e_{2^{r+1}}\right) \ldots e_{2^{k}}\right) e_{2^{i}} & =(-1)^{k-r+3} e_{T+1},
\end{aligned}
$$

where $T=2^{r}+2^{r+1}+\ldots+2^{k}+2^{i}$ and

$$
e_{1} e_{2^{i}}=e_{2^{i}+1} .
$$


Proof. From Remark 2.1, it results that we can use Theorem 1.2 for $\gamma_{1}, \gamma_{2}, \ldots, \gamma_{t}$ arbitrary. From Remark 1.1, it results $T=2^{r}+2^{r+1}+\ldots+2^{k}+2^{i}$. For $T$, we have the binary decomposition

$$
T_{2}=1 \underbrace{00 \ldots 0}_{i-k-1} \underbrace{111 \ldots 10 \ldots 0}_{k-r+1} .
$$

Using the same remark, we obtain $e_{2^{r}} e_{2^{r+1}}=\gamma_{n}(\underbrace{01 \ldots 0}_{r+2}, \underbrace{10 \ldots 0}_{r+2}) e_{2^{r}+2^{r+1}}^{10 \text { We }}$ "shuffling" $\underbrace{01 \ldots 0}_{r+2}$ and $\underbrace{10 \ldots 0}_{r+2}$ and we obtain $0110 \underbrace{0000 \ldots 0000}_{r \text { pairs }}$. Starting with $A_{0}$, it results:

$$
A_{0} \stackrel{01}{\rightarrow} A \stackrel{10}{\rightarrow} C
$$

then $\gamma_{n}(\underbrace{01 \ldots 0}_{r+2}, \underbrace{10 \ldots 0}_{r+2})=1$ and $e_{2^{r}} e_{2^{r+1}}=e_{2^{r}+2^{r+1}}$.

We compute $\left(e_{2^{r}} e_{2^{r+1}}\right) e_{2^{r+2}}$. We obtain

$$
\left(e_{2^{r}} e_{2^{r+1}}\right) e_{2^{r+2}}=e_{2^{r}+2^{r+1}} e_{2^{r+2}}=\gamma_{n}(\underbrace{011 \ldots 0}_{r+3}, \underbrace{10 \ldots 0}_{r+3}) e_{2^{r}+2^{r+1}+2^{r+2}}^{10 .}
$$

Shuffling $\underbrace{011 \ldots 0}_{r+3}$ and $\underbrace{10 \ldots 0}_{r+3}$, we get $011010 \underbrace{0000 \ldots 0000}_{r \text { pairs }}$. Starting with $A_{0}$, it results: $A_{0} \stackrel{01}{\rightarrow} A \stackrel{10}{\rightarrow} C \stackrel{10}{\rightarrow}-C$, then

$$
\gamma_{n}(\underbrace{011 \ldots 0}_{r+3}, \underbrace{10 \ldots 0}_{r+3})=-1,
$$

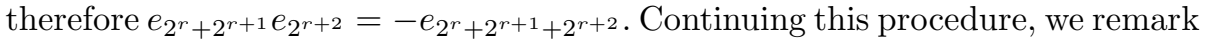
that the number of "1" in the "shuffling" obtained influences the sign. Since $T=2^{r}+2^{r+1}+\ldots+2^{k}+2^{i}$ has binary decomposition

$$
T_{2}=1 \underbrace{00 \ldots 0111 \ldots 10 \ldots 0}_{i-k-1 k-r+1},
$$

in which we have $k-r+2$ elements equal with 1 , we obtain relation (8). In the same way it results relations (9) and (10).

Proposition 2.3. With the same notations as in Proposition 2.2, for the algebra $A_{t}=\left(\frac{-1, \ldots,-1}{\mathbb{R}}\right)$, we have:

\begin{tabular}{l|ll}
$\cdot$ & $e_{T}$ & $e_{T+1}$ \\
\hline$e_{T_{1}}$ & $(-1)^{k-r+1} e_{2^{i}}$ & $-(-1)^{k-r+1} e_{2^{i}+1}$ \\
$e_{T_{1}+1}$ & $-(-1)^{k-r+1} e_{2^{i}+1}$ & $-(-1)^{k-r+1} e_{2^{i}}$
\end{tabular}


for $r<k$, where $T=2^{r}+2^{r+1}+\ldots+2^{k}+2^{i}, T_{1}=2^{r}+2^{r+1}+\ldots+2^{k}$ and

\begin{tabular}{l|ll} 
& $e_{T}$ & $e_{T+1}$ \\
\hline$e_{2^{k}}$ & $e_{M}$ & $-e_{M+1}$ \\
$e_{2^{k}+1}$ & $-e_{M+1}$ & $-e_{M}$
\end{tabular}

where $M=2^{k}+2^{i}$.

Proof. Case 1: $r<k$. We compute $e_{T_{1}} e_{T}$. We have $e_{T_{1}} e_{T}=\gamma(s, q) e_{M}$, where $s, q$ are the binary decomposition of $T_{1}$ and $T$. The binary decomposition of $M$ is $M_{2}=T_{1} \otimes T$. It results $M=2^{i}$,

$$
s=\underbrace{00 \ldots 0111 \ldots 10 \ldots 0}_{i-k}, \quad q=\underbrace{100 \ldots 0111 \ldots 10 \ldots 0}_{k-r+1} .
$$

By "shuffling" $s \otimes q$, we obtain

$$
\underbrace{010000 \ldots 00}_{(i-k) \text { pairs }} \underbrace{111111 \ldots 11}_{(k-r+1) \text { pairs }} \underbrace{0000 \ldots 0000}_{r \text { pairs }} .
$$

Starting with $A_{0}$, we get:

$$
\underbrace{A_{0} \stackrel{01}{\rightarrow} A \stackrel{00}{\rightarrow} \ldots \stackrel{00}{\rightarrow} A \stackrel{11}{\rightarrow}-C \stackrel{11}{\rightarrow} C \stackrel{11}{\rightarrow}-C \stackrel{11}{\rightarrow} C \stackrel{11}{\rightarrow} \ldots \stackrel{11}{\rightarrow}(-1)^{k-r+1} C \stackrel{00}{\rightarrow} \ldots \stackrel{00}{\rightarrow}(-1)^{k-r+1} C}_{i-k} .
$$

Therefore $\gamma(s, q)=(-1)^{k-r+1}$.

Now, we compute $e_{T_{1}} e_{T+1}$. For this, we will "shuffling" $\underbrace{00 \ldots 0111 \ldots 10 \ldots 0}_{i-k}$ with

$\underbrace{100 \ldots 0}_{i-k} \underbrace{111 \ldots 10 \ldots 1}_{k-r+1}$. It results

$$
\underbrace{010000 \ldots 00}_{(i-k) \text { pairs }} \underbrace{111111 \ldots 11}_{(k-r+1) \text { pairs }} \underbrace{0000 \ldots 0001}_{r \text { pairs }} \text {. }
$$

Starting with $A_{0}$, we get:

$\underbrace{A_{0} \stackrel{01}{\rightarrow} A \stackrel{00}{\rightarrow} \ldots \stackrel{00}{\rightarrow} A \stackrel{11}{\rightarrow}-C \stackrel{11}{\rightarrow} C \stackrel{11}{\rightarrow}-C \stackrel{11}{\rightarrow} C \stackrel{11}{\rightarrow} \ldots \stackrel{11}{\rightarrow}(-1)^{k-r+1} C \stackrel{00}{\rightarrow} \ldots \stackrel{01}{\rightarrow}-(-1)^{k-r+1} C}_{i-k}$.

For $e_{T_{1}+1} e_{T}$, "shuffling" $\underbrace{00 \ldots 0111 \ldots 10 \ldots 1}_{i-k}$ with $\underbrace{100 \ldots 0111 \ldots 10 \ldots 0}_{i-k}$, it results

$$
\underbrace{010000 \ldots 00}_{(i-k) \text { pairs }} \underbrace{110101 \ldots 01}_{(k-r+1) \text { pairs }} \underbrace{0000 \ldots 0010}_{r \text { pairs }} .
$$

Starting with $A_{0}$,we get:

$\underbrace{A_{0} \stackrel{01}{\rightarrow} A \stackrel{00}{\rightarrow} \ldots \stackrel{00}{\rightarrow} A \stackrel{11}{\rightarrow}-C \stackrel{11}{\rightarrow} C \stackrel{11}{\rightarrow}-C \stackrel{11}{\rightarrow} C \rightarrow \ldots \stackrel{11}{\rightarrow}(-1)^{k-r+1} C \stackrel{00}{\rightarrow} \ldots \stackrel{10}{\rightarrow}-(-1)^{k-r+1} C}_{i-k}$. 
For $e_{T_{1}+1} e_{T+1}$, we compute first $\left(T_{1}+1\right) \otimes(T+1)$. We obtain:

$$
\begin{gathered}
\left(2^{r}+2^{r+1}+\ldots+2^{k}+1\right) \otimes\left(2^{r}+2^{r+1}+\ldots+2^{k}+2^{i}+1\right)= \\
=(\underbrace{0 \ldots \ldots 0111 \ldots 10 \ldots 1}_{i-k}) \otimes(\underbrace{100 \ldots 0111 \ldots 10 \ldots 1}_{k-r+1})= \\
=\underbrace{10 \ldots 0000 \ldots 00 \ldots 0}_{i-k}=2_{k-r+1}^{i} .
\end{gathered}
$$

Now, "shuffling" $\underbrace{00 \ldots 0111 \ldots 10 \ldots 1}_{i-k}$ with $\underbrace{100 \ldots 0111 \ldots 10 \ldots 1}_{i-k}$, it results

$$
\underbrace{010000 \ldots 00}_{(i-k) \text { pairs }} \underbrace{110101 \ldots 01}_{(k-r+1) \text { pairs }} \underbrace{0000 \ldots 0011}_{r \text { pairs }} \text {. }
$$

Starting with $A_{0}$, we get:

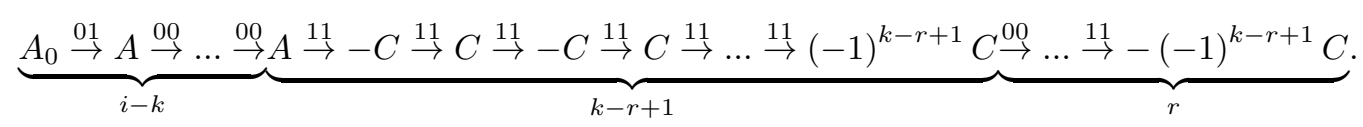

Case 2: $r=k$. We have $M=2^{k} \otimes T=2^{i}+2^{k}$. For $e_{2^{k}} e_{T}$, "shuffling" $\underbrace{00 \ldots 010 \ldots 0}_{i-k}$

with $\underbrace{100 \ldots 00 \ldots 0}_{i-k}$, it results

$$
\underbrace{010000 \ldots 00}_{(i-k) \text { pairs }} \underbrace{100000 \ldots 00}_{(k+1) \text { pairs }} \text {. }
$$

Starting with $A_{0}$, we get:

$$
\underbrace{A_{0} \stackrel{01}{\rightarrow} A \stackrel{00}{\rightarrow} \ldots . \stackrel{00}{\rightarrow}}_{i-k} \underbrace{\stackrel{10}{\rightarrow} C \stackrel{00}{\rightarrow} C \stackrel{00}{\rightarrow} \ldots \stackrel{00}{\rightarrow} C}_{k+1} .
$$

For $e_{2^{k}} e_{T+1}$, "shuffling" $\underbrace{00 \ldots 010 \ldots 0}_{i-k}$ with $\underbrace{100 \ldots 00 \ldots 1}_{i-k} \underbrace{10.1}_{k+1}$, it results

$$
\underbrace{010000 \ldots 00}_{(i-k) \text { pairs }} \underbrace{100000 \ldots 01}_{(k+1) \text { pairs }} \text {. }
$$

Starting with $A_{0}$, we get:

$$
\underbrace{A_{0} \stackrel{01}{\rightarrow} A \stackrel{00}{\rightarrow}}_{i-k} \cdots \stackrel{00}{\rightarrow} \underbrace{A \stackrel{10}{\rightarrow} C \stackrel{00}{\rightarrow} C \stackrel{00}{\rightarrow} \ldots \stackrel{01}{\rightarrow}-C}_{k+1}
$$


etc.

Proposition 2.4. Let $A_{t}=\left(\frac{\gamma_{1}, \ldots, \gamma_{t}}{\mathbb{R}}\right)$ be an algebra obtained by the CayleyDickson process. For any $x_{1}, x_{2}, \ldots, x_{t} \in \mathbb{R}-\{0\}$, we have that

$$
\left(\frac{\gamma_{1}, \ldots, \gamma_{t}}{\mathbb{R}}\right) \simeq\left(\frac{\gamma_{1} x_{1}^{2}, \ldots, \gamma_{t} x_{t}^{2}}{\mathbb{R}}\right)
$$

Proof. Let $A_{t}=\left(\frac{\gamma_{1}, \ldots, \gamma_{t}}{\mathbb{R}}\right)$ with the basis $\left\{e_{0}=1, e_{1}, \ldots, e_{n-1}\right\}, n=2^{t}$ and let $A_{t}^{\prime}=\left(\frac{\gamma_{1} x_{1}^{2}, \ldots, \gamma_{t} x_{t}^{2}}{\mathbb{R}}\right)$ with the basis $\left\{e_{0}^{\prime}=1, e_{1}^{\prime}, \ldots, e_{n-1}^{\prime}\right\}$ such that $\left(e_{i}^{\prime}\right)^{2}=$ $\gamma_{i} x_{i}^{2}, i \in\{1,2, \ldots, n-1\}$. We remark that $\left(x_{i} e_{i}\right)^{2}=x_{i}^{2} \gamma_{i}$ and from here, it results that the map $\tau: A_{t}^{\prime} \rightarrow A_{t}, \tau\left(e_{i}^{\prime}\right)=e_{i} x_{i}$ is an algebra isomorphism.

The above proposition generalized Proposition 1.1, p. 52 from [5].

Remark 2.5. From Proposition 2.4, it results that for each $n=2^{t}$ there are only $n$ non-isomorphic algebras $A_{t}$. These algebras are of the form $A_{t}=$ $\left(\frac{\gamma_{1}, \ldots, \gamma_{t}}{\mathbb{R}}\right)$, with $\gamma_{1}, \ldots, \gamma_{t} \in\{-1,1\}$.

Definition 2.6. Let $\left\{e_{0}=1, e_{1}, \ldots, e_{n-1}\right\}$ be a basis in $A_{t}=\left(\frac{\gamma_{1}, \ldots, \gamma_{t}}{\mathbb{R}}\right)$, $n=2^{t}$. To domain $\Omega \subset \mathbb{R}^{2^{t}-1}$ we will associate the domain $\Omega_{\zeta}:=\{\zeta=$ $\left.x_{1} e_{1}+\ldots+x_{n-1} e_{n-1}:\left(x_{1}, x_{2}, \ldots, x_{n-1}\right) \in \Omega\right\}$ included in $A_{t}$.

Consider a function $\Phi: \Omega_{\zeta} \rightarrow A_{t}$ of the form

$$
\Phi(\zeta)=\sum_{k=1}^{n-1} \Phi_{k}\left(x_{1}, x_{2}, \ldots, x_{n-1}\right) e_{k},
$$

where $\left(x_{1}, x_{2}, \ldots, x_{n-1}\right) \in \Omega$ and $\Phi_{k}: \Omega \rightarrow \mathbb{R}$.

We say that a function of the form (13) is left $A_{t}$-holomorphic in a domain $\Omega_{\zeta}$ if the first partial derivatives $\partial \Phi_{k} / \partial x_{k}$ exist in $\Omega$ and the following equality is fulfilled in every point of $\Omega_{\zeta}$ :

$$
D[\Phi](\zeta)=\sum_{k=1}^{2^{t}-1} e_{k} \frac{\partial \Phi}{\partial x_{k}}=0 .
$$

The operator $D$ is called Dirac operator. Note that if $A_{t}$ is the generalized quaternion algebra, then the left $A_{t}$-holomorphic functions is also called hyperholomorphic. We also note that every hyperholomorphic function $\Phi$ in a domain $\Omega_{\zeta}$ is a solution of the equation

$$
\gamma_{1} \frac{\partial^{2} \Phi}{\partial x_{1}^{2}}+\gamma_{2} \frac{\partial^{2} \Phi}{\partial x_{2}^{2}}+\gamma_{1} \gamma_{2} \frac{\partial^{2} \Phi}{\partial x_{3}^{2}}=0
$$

Remark 2.7. Let $\mathbb{H}\left(\gamma_{1}, \gamma_{2}\right)$ be the generalized quaternion algebra with the basis $\left\{1, e_{1}, e_{2}, e_{3}\right\}, \gamma_{1}>0, \gamma_{2}>0$ and $\mathbb{H}(-1,-1)$ be the usual quaternion division algebra with the basis $\{1, i, j, k\}$. Let $\Omega$ be a domain in $\mathbb{R}^{3}$, and let 
$\Omega_{\zeta}:=\{\zeta=x i+y j+z k:(x, y, z) \in \Omega\}$ be a corresponding domain in $\mathbb{H}(-1,-1)$. The function $\Phi: \Omega_{\zeta} \rightarrow \mathbb{H}(-1,-1)$ of the form

$$
\Phi(\zeta)=u_{1}(x, y, z)+u_{2}(x, y, z) i+u_{3}(x, y, z) j+u_{4}(x, y, z) k .
$$

is hyperholomorphic in the domain $\Omega$ if

$$
D[\Phi](\zeta)=i \frac{\partial \Phi}{\partial x}+j \frac{\partial \Phi}{\partial y}+k \frac{\partial \Phi}{\partial z}=0 .
$$

For another domain $\Delta \subset \mathbb{R}^{3}$, we associate the domain $\Delta_{\widetilde{\zeta}}:=\left\{\widetilde{\zeta}=\widetilde{x} e_{1}+\widetilde{y} e_{2}+\right.$ $\left.\widetilde{z} e_{3}:(\widetilde{x}, \widetilde{y}, \widetilde{z}) \in \Delta\right\}$ in the algebra $\mathbb{H}\left(\gamma_{1}, \gamma_{2}\right)$. The Dirac operator in $\mathbb{H}\left(\gamma_{1}, \gamma_{2}\right)$, denoted by $\widetilde{D}$, is

$$
\widetilde{D}:=e_{1} \frac{\partial}{\partial \widetilde{x}}+e_{2} \frac{\partial}{\partial \widetilde{y}}+e_{3} \frac{\partial}{\partial \widetilde{z}} .
$$

The elements of bases in $\mathbb{H}(-1,-1)$ and $\mathbb{H}\left(\gamma_{1}, \gamma_{2}\right)$ satisfy the following equalities:

$$
e_{1}=i \sqrt{\gamma_{1}}, \quad e_{2}=j \sqrt{\gamma_{2}}, \quad e_{3}=k \sqrt{\gamma_{1} \gamma_{2}} .
$$

Now we establish a connection between hyperholomorphic functions in the algebras $\mathbb{H}(-1,-1)$ and $\mathbb{H}\left(\gamma_{1}, \gamma_{2}\right)$, where $\gamma_{1}>0, \gamma_{2}>0$. For this, we denote

$$
x=\frac{1}{\sqrt{\gamma_{1}}} \widetilde{x}, \quad y=\frac{1}{\sqrt{\gamma_{2}}} \widetilde{y}, \quad z=\frac{1}{\sqrt{\gamma_{1} \gamma_{2}}} \widetilde{z} .
$$

These relations give us the operator equalities:

$$
\frac{\partial}{\partial \widetilde{x}}=\frac{1}{\sqrt{\gamma_{1}}} \frac{\partial}{\partial x}, \quad \frac{\partial}{\partial \widetilde{y}}=\frac{1}{\sqrt{\gamma_{2}}} \frac{\partial}{\partial y}, \quad \frac{\partial}{\partial \widetilde{z}}=\frac{1}{\sqrt{\gamma_{1} \gamma_{2}}} \frac{\partial}{\partial z} .
$$

Now, using relations (14) and (15), we obtain

$$
\begin{gathered}
\widetilde{D}[\Phi](\widetilde{\zeta})=e_{1} \frac{\partial \Phi}{\partial \widetilde{x}}+e_{2} \frac{\partial \Phi}{\partial \widetilde{y}}+e_{3} \frac{\partial \Phi}{\partial \widetilde{z}}= \\
=i \frac{\partial \Phi}{\partial x} \frac{1}{\sqrt{\gamma_{1}}} \sqrt{\gamma_{1}}+j \frac{\partial \Phi}{\partial y} \frac{1}{\sqrt{\gamma_{2}}} \sqrt{\gamma_{2}}+k \frac{\partial \Phi}{\partial z} \frac{1}{\sqrt{\gamma_{1} \gamma_{2}}} \sqrt{\gamma_{1} \gamma_{2}}= \\
=i \frac{\partial \Phi}{\partial x}+j \frac{\partial \Phi}{\partial y}+k \frac{\partial \Phi}{\partial z}=D[\Phi](\zeta)=0 .
\end{gathered}
$$

Using the above notations, we obtain the following theorem:

Theorem 2.8. Let $\Omega$ be an arbitrary domain in $\mathbb{R}^{3}$ and $\Delta$ be a domain in $\mathbb{R}^{3}$ such that the coordinates of the corresponding points $\zeta=x i+y j+z k \in \Omega_{\zeta}$ and $\widetilde{\zeta}=\widetilde{x} e_{1}+\widetilde{y} e_{2}+\widetilde{z} e_{3} \in \Delta_{\widetilde{\zeta}}$ satisfy the following relations:

$$
x=\frac{1}{\sqrt{\operatorname{sign}\left(\gamma_{1}\right) \gamma_{1}}} \widetilde{x}, \quad y=\frac{1}{\sqrt{\operatorname{sign}\left(\gamma_{2}\right) \gamma_{2}}} \widetilde{y}, \quad z=\frac{1}{\sqrt{\operatorname{sign}\left(\gamma_{1}\right) \operatorname{sign}\left(\gamma_{2}\right) \gamma_{1} \gamma_{2}}} \widetilde{z},
$$


where sign $(a)$ is the sign of the non-zero real number a. Then if the function $\Phi: \Omega_{\zeta} \rightarrow \mathbb{H}\left(\operatorname{sign}\left(\gamma_{1}\right)\right.$, sign $\left.\left(\gamma_{2}\right)\right)$ is hyperholomorphic in the domain $\Omega_{\zeta}$, then the same function $\Phi$, of $\widetilde{\zeta}$, is hyperholomorphic in the domain $\Delta_{\widetilde{\zeta}} \in \mathbb{H}\left(\gamma_{1}, \gamma_{2}\right)$. The converse is also true.

Proof. Since $e_{1}=i \sqrt{\operatorname{sign}\left(\gamma_{1}\right) \gamma_{1}}, e_{2}=j \sqrt{\operatorname{sign}\left(\gamma_{2}\right) \gamma_{2}}$, $e_{3}=k \sqrt{\operatorname{sign}\left(\gamma_{1}\right) \operatorname{sign}\left(\gamma_{2}\right) \gamma_{1} \gamma_{2}}$, the result directly follows from Remark 2.7.

Remark 2.9. (i) The above Theorem tell us that for studying hyperholomorphic functions in generalized quaternion algebras $\mathbb{H}\left(\gamma_{1}, \gamma_{2}\right)$ it is suffices to consider hyperholomorphic functions only in the algebras $\mathbb{H}\left(\operatorname{sign}\left(\gamma_{1}\right), \operatorname{sign}\left(\gamma_{2}\right)\right)$.

(ii) The result similar to the previous remark was established in the paper [8] (Theorem 5) in a three-dimensional commutative associative algebra.

Theorem 2.10. Let $A_{t}=\left(\frac{\gamma_{1}, \ldots, \gamma_{t}}{\mathbb{R}}\right)$ be a generalized Cayley-Dickson algebra. Let $\Omega$ be an arbitrary domain in $\mathbb{R}^{2^{t}-1}$ and $\Delta$ be a domain in $\mathbb{R}^{2^{t}-1}$ such that the coordinates of the corresponding points $\zeta=x_{1} e_{1}+\ldots+x_{2^{t}-1} e_{2^{t}-1} \in \Omega_{\zeta}$ and $\widetilde{\zeta}=\widetilde{x}_{1} \widetilde{e}_{1}+\widetilde{x}_{2} \widetilde{e}_{2}+\ldots+\widetilde{x}_{2^{t}-1} \widetilde{e}_{2^{t}-1} \in \Delta_{\widetilde{\zeta}}$ satisfy the following relations

$$
\begin{gathered}
x_{1}=\frac{1}{\sqrt{\operatorname{sign}\left(\gamma_{1}\right) \gamma_{1}}} \widetilde{x}_{1}, \quad x_{2}=\frac{1}{\sqrt{\operatorname{sign}\left(\gamma_{2}\right) \gamma_{2}}} \widetilde{x}_{2}, \ldots \\
\ldots, x_{n}=\frac{1}{\sqrt{\operatorname{sign}\left(\gamma_{1}\right) \ldots \operatorname{sign}\left(\gamma_{t}\right) \gamma_{1} \ldots \gamma_{t}}} \widetilde{x}_{n} .
\end{gathered}
$$

If the function $\Phi: \Omega_{\zeta} \rightarrow\left(\frac{\operatorname{sign}\left(\gamma_{1}\right), \ldots, \operatorname{sign}\left(\gamma_{t}\right)}{\mathbb{R}}\right)$ is left $A_{t}$-holomorphic in the domain $\Omega_{\zeta}$, then the same function $\Phi$, but depending of $\widetilde{\zeta}$ is left $A_{t}$-holomorphic in the domain $\Delta_{\widetilde{\zeta}} \in A_{t}$. The converse is also true.

Proof. Let $\left\{1, e_{1}, \ldots, e_{n-1}\right\}$ be a basis in $\left(\frac{\operatorname{sign}\left(\gamma_{1}\right), \ldots, \operatorname{sign}\left(\gamma_{t}\right)}{\mathbb{R}}\right)$ and $\left\{1, \widetilde{e}_{1}, \ldots, \widetilde{e}_{n-1}\right\}$ be a basis in $A_{t}=\left(\frac{\gamma_{1}, \ldots, \gamma_{t}}{\mathbb{R}}\right)$.

Since

$$
\begin{gathered}
\widetilde{e}_{1}=e_{1} \sqrt{\operatorname{sign}\left(\gamma_{1}\right) \gamma_{1}}, \quad \widetilde{e}_{2}=e_{2} \sqrt{\operatorname{sign}\left(\gamma_{2}\right) \gamma_{2}}, \ldots, \\
\ldots, \widetilde{e}_{n-1}=e_{n-1} \sqrt{\operatorname{sign}\left(\gamma_{1}\right) \ldots \operatorname{sign}\left(\gamma_{t}\right) \gamma_{1} \ldots \gamma_{t}}
\end{gathered}
$$

the result is obtained from a simple computation as in Remark 2.7.

Remark 2.11. Using above Theorem, it is obvious that, for studying left $A_{t}$-holomorphic functions in generalized Cayley-Dickson algebras $A_{t}=\left(\frac{\gamma_{1}, \ldots, \gamma_{t}}{\mathbb{R}}\right)$ it is suffices to consider left $A_{t}$-holomorphic functions only in the algebras $\left(\frac{\operatorname{sign}\left(\gamma_{1}\right), \ldots, \operatorname{sign}\left(\gamma_{t}\right)}{\mathbb{R}}\right)$.

Now we consider another class of differentiable functions. Let $A_{t}=\left(\frac{\gamma_{1}, \ldots, \gamma_{t}}{\mathbb{R}}\right)$, with $\gamma_{1}=\ldots=\gamma_{t}=-1$, and the domain $\Omega \subset \mathbb{R}^{2^{t}}$. We denote with $\Omega_{\zeta}:=\{\zeta=$ $\left.x_{0}+x_{1} e_{1}+\ldots+x_{n-1} e_{n-1}:\left(x_{0}, x_{1}, \ldots, x_{n-1}\right) \in \Omega\right\}$ a domain in $A_{t}$. This domain is called congruent with the domain $\Omega$. 
We consider a function $\Phi: \Omega_{\zeta} \rightarrow A_{t}$ of the form

$$
\Phi(\zeta)=\sum_{k=0}^{n-1} \Phi_{k}\left(x_{0}, x_{1}, \ldots, x_{n-1}\right) e_{k},
$$

where $\left(x_{0}, x_{1}, \ldots, x_{n-1}\right) \in \Omega$ and $\Phi_{k}: \Omega \rightarrow \mathbb{R}$.

We say that a function of the form (16) is left $A_{t}$-hyperholomorphic in a domain $\Omega_{\zeta}$ if the first partial derivatives $\partial \Phi_{k} / \partial x_{k}$ exist in $\Omega$ and the following equality is fulfilled in every point of $\Omega_{\zeta}$ :

$$
\sum_{k=0}^{2^{t}-1} e_{k} \frac{\partial \Phi}{\partial x_{k}}=0 .
$$

In the following, we will provide an algorithm to constructing a left $A_{t}$-hyperholomorphic functions. Using the above notations, let $v(x, y)$ be a rational function defined in a domain $G \subset \mathbb{R}^{2}$. In the following, using some ideas given in Theorem 3 from [12, we will give an example of left $A_{t}$-hyperholomorphic function, for all $t \geq 1, t \in \mathbb{N}$. For this, we consider the following functions:

$$
\begin{gathered}
\phi_{1}=x_{0}+e_{1} x_{1}, \quad \phi_{2}=\frac{1}{e_{1}}\left(x_{0}+e_{1} x_{1}\right), \\
\rho_{2 s-1}=x_{2 s}-e_{1} x_{2 s+1}, \quad \rho_{2 s}=-\frac{1}{e_{1}}\left(x_{2 s}-e_{1} x_{2 s+1}\right), \quad s \in\left\{1,2, \ldots, 2^{t-1}-1\right\}, \\
F_{t}(\zeta)=v\left(\phi_{1}, \phi_{2}\right)+v\left(\rho_{1}, \rho_{2}\right) e_{2}+v\left(\rho_{3}, \rho_{4}\right) e_{4}+\left[v\left(\rho_{5}, \rho_{6}\right) e_{2}\right] e_{4}+ \\
+v\left(\rho_{7}, \rho_{8}\right) e_{8}+\left(v\left(\rho_{9}, \rho_{10}\right) e_{2}\right) e_{8}+\left(v\left(\rho_{11}, \rho_{12}\right) e_{4}\right) e_{8}+\left[\left(v\left(\rho_{13}, \rho_{14}\right) e_{2}\right) e_{4}\right] e_{8}+\ldots \\
\left.\left.\ldots+\sum_{i=4}^{t-1}\left(\sum_{k=1}^{i}\left(\sum_{r=1}^{k-1} v\left(\rho_{M_{r k i}-1}, \rho_{M_{r k i}}\right) e_{2^{r}}\right) e_{2^{r+1}} \ldots\right) e_{2^{k}}\right) e_{2^{i}}\right)+\sum_{i=1}^{t-1}\left(v\left(\rho_{2^{i}-1}, \rho_{2^{i}}\right) e_{2^{i}}\right),
\end{gathered}
$$

where $M_{r k i}=2^{r}+2^{r+1}+\ldots+2^{k}+2^{i}$.

It results

$$
\begin{gathered}
F_{t}(\zeta)=v\left(\phi_{1}, \phi_{2}\right)+ \\
\left.+\sum_{i=1}^{t-1}\left(\sum_{k=1}^{i}\left(\sum_{r=1}^{k-1} v\left(\rho_{M_{r k i}-1}, \rho_{M_{r k i}}\right) e_{2^{r}}\right) e_{2^{r+1} \ldots} \ldots e_{2^{k}}\right) e_{2^{i}}\right)+\sum_{i=1}^{t-1}\left(v\left(\rho_{2^{i}-1}, \rho_{2^{i}}\right) e_{2^{i}}\right),
\end{gathered}
$$

or

$$
\begin{gathered}
F_{t}(\zeta)=F_{t-1}(\zeta)+ \\
\left.+\left(\sum_{k=1}^{t-2}\left(\sum_{r=1}^{k-1} v\left(\rho_{M_{r k(t-1)}-1}, \rho_{M_{r k(t-1)}}\right) e_{2^{r}}\right) e_{2^{r+1} \ldots} \ldots e_{2^{k}}\right) e_{2^{t-1}}\right)+v\left(\rho_{2^{t-1}-1}, \rho_{2^{t-1}}\right) e_{2^{t-1}}
\end{gathered}
$$


We denote with $\mathbb{C}_{2 s}$ the "complex" planes $\left\{x_{2 s}+e_{1} x_{2 s+1}: x_{2 s}, x_{2 s+1} \in \mathbb{R}\right\}$ and with $D_{2 s}:=\left\{\left(x_{2 s}, x_{2 s+1}\right): x_{2 s}+e_{1} x_{2 s+1} \in \mathbb{C}_{2 s}\right\}, s \in\left\{0,1,2, \ldots, 2^{t-1}-1\right\}$ the Euclidian planes. Let $G_{2 s}$ be a domains in $\mathbb{C}_{2 s}$ and let $\widetilde{G}_{2 s}$ be the corresponded domains in $D_{2 s}$. We have the following theorem:

Theorem 2.12. With the above notations, we consider the functions $v\left(\phi_{1}, \phi_{2}\right)$ and $v\left(\rho_{2 s-1}, \rho_{2 s}\right)$ defined in the corresponding domains $G_{0} \subset \mathbb{C}_{0}$ and $G_{2 s} \subset$ $\mathbb{C}_{2 s}, s \in\left\{1,2, \ldots, 2^{t-1}-1\right\}$. Then the map $F_{t}(\zeta)$ is a left $A_{t}$-hyperholomorphic function in the domain $\Theta \subset A_{t}$ which is congruent with the domain $\widetilde{G}_{0} \times \widetilde{G}_{2} \times$ $\widetilde{G}_{4} \times \ldots \times \widetilde{G}_{2^{t-1}-1} \subset \mathbb{R}^{2^{t}}$, for $t \geq 1$.

Proof. For $t=1$, we have $F_{1}(\zeta)=v\left(\phi_{1}, \phi_{2}\right)$, which is an holomorphic function in $D_{0} \subset \mathbb{C}_{0}$, as we can see in [12, Theorem 3 .

For $t=2$, we obtain $F_{2}(\zeta)=v\left(\phi_{1}, \phi_{2}\right)+v\left(\rho_{1}, \rho_{2}\right) e_{2}$ and for $t=3$, we get $F_{3}(\zeta)=v\left(\phi_{1}, \phi_{2}\right)+v\left(\rho_{1}, \rho_{2}\right) e_{2}+v\left(\rho_{3}, \rho_{4}\right) e_{4} . F_{2}(\zeta)$ and $F_{3}(\zeta)$ are hyperholomorphic, respectively octonionic hyperholomorphic function, from Remark 2.1 and Theorem 3 from 12 .

For $t \geq 4$, using induction steps, supposing that $F_{t-1}(\zeta)$ is a left $A_{t-1^{-}}$ hyperholomorphic function, we will prove that $F_{t}(\zeta)$ is $A_{t}$-hyperholomorphic. That means $D\left[F_{t}\right]=0$. From relations (6) and (7), we have that

$$
\begin{aligned}
D\left[F_{t}\right]= & \sum_{k=0}^{2^{t}-1} e_{k} \frac{\partial F_{t}}{\partial x_{k}}=\sum_{k=0}^{2^{t-1}-1} e_{k} \frac{\partial F_{t}}{\partial x_{k}}+\sum_{k=2^{t-1}}^{2^{t}-1} e_{k} \frac{\partial F_{t}}{\partial x_{k}}= \\
& =D\left[F_{t-1}\right]+e_{2^{t-1}} \sum_{k=0}^{2^{t-1}-1} \bar{e}_{k} \frac{\partial F_{t}}{\partial x_{k+2^{t-1}}} .
\end{aligned}
$$

From induction steps, we obtain $D\left[F_{t-1}\right]=0$. We will prove that $\sum_{k=0}^{2^{t-1}-1} \bar{e}_{k} \frac{\partial F_{t}}{\partial x_{2^{t-1}+k}}=$ 0 . This sum has $2^{t-1}$ terms. First two terms are:

$$
\begin{gathered}
\left(\frac{\partial F_{t}}{\partial x_{2^{t-1}}}-e_{1} \frac{\partial F_{t}}{\partial x_{2^{t-1}+1}}\right)= \\
=\frac{\partial v}{\partial \rho_{2^{t-1}-1}} \frac{\partial \rho_{2^{t-1}-1}}{\partial x_{2^{t-1}}}+\frac{\partial v}{\partial \rho_{2^{t-1}}} \frac{\partial \rho_{2^{t-1}}}{\partial x_{2^{t-1}}}-e_{1}\left(\frac{\partial v}{\partial \rho_{2^{t-1}-1}} \frac{\partial \rho_{2^{t-1}-1}}{\partial x_{2^{t-1}+1}}+\frac{\partial v}{\partial \rho_{2^{t-1}}} \frac{\partial \rho_{2^{t-1}}}{\partial x_{2^{t-1}}+1}\right)= \\
=\frac{\partial v}{\partial \rho_{2^{t-1}-1}}+\frac{\partial v}{\partial \rho_{2^{t-1}}}\left(\frac{-1}{e_{1}}\right)-e_{1}\left(\frac{\partial v}{\partial \rho_{2^{t-1}-1}}\left(-e_{1}\right)+\frac{\partial v}{\partial \rho_{2^{t-1}}}\right)= \\
=\frac{\partial v}{\partial \rho_{2^{t-1}-1}}+\frac{\partial v}{\partial \rho_{2^{t-1}}} e_{1}-\frac{\partial v}{\partial \rho_{2^{t-1}-1}}-e_{1} \frac{\partial v}{\partial \rho_{2^{t-1}}}=0 .
\end{gathered}
$$


Since $e_{1}^{2}=\gamma_{1}, \gamma_{1}^{2}=1, \frac{\partial v}{\partial \rho_{2^{t-1}-1}}$ and $\frac{\partial v}{\partial \rho_{2^{t-1}}}$ can be written as $a_{2^{t-1}-1}(\zeta)+$ $b_{2^{t-1}-1}(\zeta) e_{1}$, respectively $a_{2^{t-1}}(\zeta)+b_{2^{t-1}}(\zeta) e_{1}$ where $a_{2^{t-1}-1}(\zeta), b_{2^{t-1}-1}(\zeta)$, $a_{2^{t-1}}(\zeta), b_{2^{t-1}}(\zeta)$ are real valued functions.

Case 1: $r<k$. In the general case, we denote $T=2^{r}+2^{r+1}+\ldots+2^{k}+2^{t-1}$ and $T_{1}=2^{r}+2^{r+1}+\ldots+2^{k}$, for $r<k$. We will compute the terms

$$
-e_{T_{1}} \frac{\partial F_{t}}{\partial x_{T}}-e_{T_{1}+1} \frac{\partial F_{t}}{\partial x_{T+1}} .
$$

We compute first $\frac{\partial F_{t}}{\partial x_{T}}$. It results

$$
\begin{aligned}
\frac{\partial F_{t}}{\partial x_{T}}= & \left.\left.\left(\ldots\left(\frac{\partial v}{\partial \rho_{T-1}} \frac{\partial \rho_{T-1}}{\partial x_{T}}+\frac{\partial v}{\partial \rho_{T}} \frac{\partial \rho_{T}}{\partial x_{T}}\right) e_{2^{r}}\right) e_{2^{r+1}}\right) \ldots e_{2^{k}}\right) e_{2^{t-1}}= \\
= & \left.\left.\left(\ldots\left(\frac{\partial v}{\partial \rho_{T-1}}+\frac{\partial v}{\partial \rho_{T}} \frac{-1}{e_{1}}\right) e_{2^{r}}\right) e_{2^{r+1}}\right) \ldots e_{2^{k}}\right) e_{2^{t-1}}= \\
& \left.\left.=\left(\ldots\left(\frac{\partial v}{\partial \rho_{T-1}}+\frac{\partial v}{\partial \rho_{T}} e_{1}\right) e_{2^{r}}\right) e_{2^{r+1}}\right) \ldots e_{2^{k}}\right) e_{2^{t-1}} .
\end{aligned}
$$

Since we can write $\frac{\partial v}{\partial \rho_{T-1}}$ under the form $a_{T-1}(\zeta)+b_{T-1}(\zeta) e_{1}$ and $\frac{\partial v}{\partial \rho_{T}}$ under the form $a_{T}(\zeta)+b_{T}(\zeta) e_{1}$, where $a_{T-1}, b_{T-1}, a_{T}, b_{T}$ are real valued functions, using Proposition 2.2, we obtain:

$$
\begin{gathered}
\left.\left.\frac{\partial F_{t}}{\partial x_{T}}=\left(\ldots\left(\frac{\partial v}{\partial \rho_{T-1}}+\frac{\partial v}{\partial \rho_{T}} e_{1}\right) e_{2^{r}}\right) e_{2^{r+1}}\right) \ldots e_{2^{k}}\right) e_{2^{t-1}}= \\
\left.\left.\left.=\left(\ldots\left(a_{T-1}(\zeta) e_{2^{r}}\right) e_{2^{r+1}}\right) \ldots e_{2^{k}}\right) e_{2^{t-1}}+\left(\ldots\left(b_{T-1}(\zeta) e_{1}\right) e_{2^{r}}\right) e_{2^{r+1}}\right) \ldots e_{2^{k}}\right) e_{2^{t-1}}+ \\
\left.\left.\left.\left.\left.+\left(\ldots\left(a_{T}(\zeta) e_{1}\right) e_{2^{r}}\right) e_{2^{r+1}}\right) \ldots e_{2^{k}}\right) e_{2^{t-1}}+\left(\ldots\left(b_{T}(\zeta) e_{1}\right) e_{1}\right) e_{2^{r}}\right) e_{2^{r+1}}\right) \ldots e_{2^{k}}\right) e_{2^{t-1}}= \\
=a_{T-1}(\zeta)(-1)^{k-r+2} e_{T}+b_{T-1}(\zeta)(-1)^{k-r+3} e_{T+1}+ \\
+a_{T}(\zeta)(-1)^{k-r+3} e_{T+1}-b_{T}(\zeta)(-1)^{k-r+2} e_{T} .
\end{gathered}
$$

Using Proposition 2.3, relation (11), we compute $-e_{T_{1}} \frac{\partial F_{t}}{\partial x_{T}}$.

$$
\begin{aligned}
-e_{T_{1}} \frac{\partial F_{t}}{\partial x_{T}} & =-e_{T_{1}}\left(a_{T-1}(\zeta)(-1)^{k-r+2} e_{T}+b_{T-1}(\zeta)(-1)^{k-r+3} e_{T+1}+\right. \\
& \left.+a_{T}(\zeta)(-1)^{k-r+3} e_{T+1}-b_{T}(\zeta)(-1)^{k-r+2} e_{T}\right)=
\end{aligned}
$$




$$
\begin{gathered}
=-\left(a_{T-1}(\zeta)(-1)^{k-r+2}(-1)^{k-r+1} e_{2^{i}}-b_{T-1}(\zeta)(-1)^{k-r+3}(-1)^{k-r+1} e_{2^{i}+1}\right)- \\
-\left(-a_{T}(\zeta)(-1)^{k-r+3}(-1)^{k-r+1} e_{2^{i}+1}-b_{T}(\zeta)(-1)^{k-r+2}(-1)^{k-r+1} e_{2^{i}}\right)= \\
=-\left(a_{T-1}(\zeta)(-1)^{2 k-2 r+3} e_{2^{i}}-b_{T-1}(\zeta)(-1)^{2 k-2 r+4} e_{2^{i}+1}\right)- \\
-\left(-a_{T}(\zeta)(-1)^{2 k-2 r+4} e_{2^{i}+1}-b_{T}(\zeta)(-1)^{2 k-2 r+3} e_{2^{i}}\right) .
\end{gathered}
$$

Now, we compute $\frac{\partial F_{t}}{\partial x_{T+1}}$. We obtain

$$
\begin{aligned}
\frac{\partial F_{t}}{\partial x_{T+1}}= & \left.\left.\left(\ldots\left(\frac{\partial v}{\partial \rho_{T-1}} \frac{\partial \rho_{T-1}}{\partial x_{T+1}}+\frac{\partial v}{\partial \rho_{T}} \frac{\partial \rho_{T}}{\partial x_{T+1}}\right) e_{2^{r}}\right) e_{2^{r+1}}\right) \ldots e_{2^{k}}\right) e_{2^{t-1}}= \\
& \left.\left.=\left(\ldots\left(-\frac{\partial v}{\partial \rho_{T-1}} e_{1}+\frac{\partial v}{\partial \rho_{T}}\right) e_{2^{r}}\right) e_{2^{r+1}}\right) \ldots e_{2^{k}}\right) e_{2^{t-1}} .
\end{aligned}
$$

Since we can write $\frac{\partial v}{\partial \rho_{T-1}}$ under the form $a_{T-1}(\zeta)+b_{T-1}(\zeta) e_{1}$ and $\frac{\partial v}{\partial \rho_{T}}$ under the form $a_{T}(\zeta)+b_{T}(\zeta) e_{1}$, where $a_{T-1}, b_{T-1}, a_{T}, b_{T}$ are real valued functions, using Proposition 2.2, we obtain:

$$
\begin{gathered}
\left.\left.\frac{\partial F_{t}}{\partial x_{T+1}}=\left(\ldots\left(-\frac{\partial v}{\partial \rho_{T-1}} e_{1}+\frac{\partial v}{\partial \rho_{T}}\right) e_{2^{r}}\right) e_{2^{r+1}}\right) \ldots e_{2^{k}}\right) e_{2^{t-1}}= \\
\left.\left.\left.\left.=\left(\ldots\left(-a_{T-1}(\zeta) e_{1}\right) e_{2^{r}}\right) e_{2^{r+1}}\right) \ldots e_{2^{k}}\right) e_{2^{t-1}}-\left(\ldots\left(b_{T-1}(\zeta) e_{1} e_{1}\right) e_{2^{r}}\right) e_{2^{r+1}}\right) \ldots e_{2^{k}}\right) e_{2^{t-1}}+ \\
\left.\left.\left.\left.\left.+\left(\ldots\left(a_{T}(\zeta)\right) e_{2^{r}}\right) e_{2^{r+1}}\right) \ldots e_{2^{k}}\right) e_{2^{t-1}}+\left(\ldots\left(b_{T}(\zeta) e_{1}\right)\right) e_{2^{r}}\right) e_{2^{r+1}}\right) \ldots e_{2^{k}}\right) e_{2^{t-1}}= \\
=-a_{T-1}(\zeta)(-1)^{k-r+3} e_{T+1}+b_{T-1}(\zeta)(-1)^{k-r+2} e_{T}+ \\
+a_{T}(\zeta)(-1)^{k-r+2} e_{T}+b_{T}(\zeta)(-1)^{k-r+3} e_{T+1} .
\end{gathered}
$$

Using Proposition 2.3, we compute $-e_{T_{1}+1} \frac{\partial F_{t}}{\partial x_{T+1}}$.

$$
-e_{T_{1}+1} \frac{\partial F_{t}}{\partial x_{T+1}}=-e_{T_{1}+1}\left(-a_{T-1}(\zeta)(-1)^{k-r+3} e_{T+1}+b_{T-1}(\zeta)(-1)^{k-r+2} e_{T}+\right.
$$




$$
\begin{gathered}
\left.+a_{T}(\zeta)(-1)^{k-r+2} e_{T}+b_{T}(\zeta)(-1)^{k-r+3} e_{T+1}\right)= \\
=-\left(a_{T-1}(\zeta)(-1)^{k-r+3}(-1)^{k-r+1} e_{2^{i}}-b_{T-1}(\zeta)(-1)^{k-r+2}(-1)^{k-r+1} e_{2^{i}+1}\right)- \\
-\left(-a_{T}(\zeta)(-1)^{k-r+2}(-1)^{k-r+1} e_{2^{i}+1}-b_{T}(\zeta)(-1)^{k-r+3}(-1)^{k-r+1} e_{2^{i}}\right)= \\
=-\left(a_{T-1}(\zeta)(-1)^{2 k-2 r+4} e_{2^{i}}-b_{T-1}(\zeta)(-1)^{2 k-2 r+3} e_{2^{i}+1}\right)- \\
-\left(-a_{T}(\zeta)(-1)^{2 k-2 r+3} e_{2^{i}+1}-b_{T}(\zeta)(-1)^{2 k-2 r+4} e_{2^{i}}\right)
\end{gathered}
$$

Now, we can compute $-e_{T_{1}} \frac{\partial F_{t}}{\partial x_{T}}-e_{T_{1}+1} \frac{\partial F_{t}}{\partial x_{T+1}}$. It results

$$
\begin{gathered}
-e_{T_{1}} \frac{\partial F_{t}}{\partial x_{T}}-e_{T_{1}+1} \frac{\partial F_{t}}{\partial x_{T+1}}= \\
=-\left(a_{T-1}(\zeta)(-1)^{2 k-2 r+3} e_{2^{i}}-b_{T-1}(\zeta)(-1)^{2 k-2 r+4} e_{2^{i}+1}\right)- \\
-\left(-a_{T}(\zeta)(-1)^{2 k-2 r+4} e_{2^{i}+1}-b_{T}(\zeta)(-1)^{2 k-2 r+3} e_{2^{i}}\right)- \\
-\left(a_{T-1}(\zeta)(-1)^{2 k-2 r+4} e_{2^{i}}-b_{T-1}(\zeta)(-1)^{2 k-2 r+3} e_{2^{i}+1}\right)- \\
-\left(-a_{T}(\zeta)(-1)^{2 k-2 r+3} e_{2^{i}+1}-b_{T}(\zeta)(-1)^{2 k-2 r+4} e_{2^{i}}\right)=0 .
\end{gathered}
$$

Case 2: $r=k$, we use Proposition 2.2 and Proposition 2.3, relation (12) and it easy to show that

$$
-e_{2^{k}} \frac{\partial F_{t}}{\partial x_{T}}-e_{2^{k}+1} \frac{\partial F_{t}}{\partial x_{T+1}}=0 .
$$

Remark 2.13. The above proposition generalizes Theorem 3 from [12].

\section{The Algorithm}

1) Input $t$.

2) Input functions $v, \phi_{1}, \phi_{2}$. 
3) For $i \in\{1, \ldots, t-1\}, k \in\{1, \ldots, i\}, r \in\{1, \ldots, k-1\}$, compute $M_{r k i}=$ $2^{r}+\ldots+2^{k}+2^{i}, v\left(\rho_{M_{r k i}-1}, \rho_{M_{r k i}}\right)=\alpha_{M_{r k i}}+\beta_{M_{r k i}} e_{1}$.

4) For $i \in\{1, \ldots, t-1\}, k \in\{1, \ldots, i\}, r \in\{1, \ldots, k-1\}$,

-if $r<k$, we compute

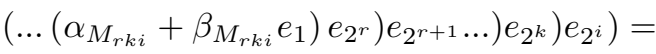

$$
\begin{aligned}
& =(-1)^{k-r+2}\left(\alpha_{M_{r k i}} e_{M_{r k i}}-\beta_{M_{r k i}} e_{M_{r k i}-1}\right)
\end{aligned}
$$

-if $r=k$, we compute

$$
\begin{gathered}
v\left(\rho_{2^{i}-1}, \rho_{2^{i}}\right) e_{2^{i}}=\left(\alpha_{2^{i}-1}+\beta_{2^{i}-1} e_{1}\right) e_{2^{i}}= \\
=\alpha_{2^{i}-1} e_{2^{i}}+\beta_{2^{i}-1} e_{2^{i}+1} .
\end{gathered}
$$

5) Output function

$$
\begin{aligned}
F_{t}(\zeta)=v\left(\phi_{1}, \phi_{2}\right)+ & \sum_{i=4}^{t-1}\left(\sum_{k=1}^{i}\left(\sum_{r=1}^{k-1}(-1)^{k-r+2}\left(\alpha_{M_{r k i}}(\zeta) e_{M_{r k i}}-\beta_{M_{r k i}}(\zeta) e_{M_{r k i}-1}\right)\right)+\right. \\
& +\sum_{i=1}^{t-1}\left(\alpha_{2^{i}-1}(\zeta) e_{2^{i}}+\beta_{2^{i}-1}(\zeta) e_{2^{i}+1}\right) .
\end{aligned}
$$

Conclusion. In this paper, we generalized the notion of left $A_{t}$-holomorphic functions from quaternions to all algebras obtained by the Cayley-Dickson process and we provided an algorithm to find examples of left $A_{t}$-hyperholomorphic functions, using the shuffling procedure given by Bales in [1].

The theory of the right $A_{t}$-holomorphic functions and the theory of the right $A_{t}$-hyperholomorphic functions are similarly to the corresponding theories for the left functions and can be easy treated, using the above ideas and procedures.

This paper is supported by the grant of CNCS (Romanian National Council of Research) PN-II-ID-WE-2012-4-169.

\section{References}

[1] Bales, J. W., A Tree for Computing the Cayley-Dickson Twist, Missouri J. Math. Sci., 21(2)(2009), 83-93. 
[2] Fueter, R., Die Funktionentheorie der Differentialgleichungen $\Delta u=0$ und $\Delta \Delta u=0$ mit vier reellen Variablen, Commet. Math. Helv., 7(1935), 307330 .

[3] Gürlebeck, K., Sprössig W., Quaternionic and Clifford calculus for physicists and engineers, John Wiley and Sons, 1997.

[4] Kravchenko, V., Shapiro M., Integral representations for spatial models of mathematical physics, Addison Wesley Longman Limited, Essex, England, 1996.

[5] Lam, T. Y., Introduction To Quadratic Forms Over Fields, Graduate Studies in Mathematics, vol 67, American Mathematical Society, Providence RI, 2005.

[6] Ludkovsky, S. V. Differentiable functions of Cayley-Dickson numbers and line integration, J. Math. Sci., 141(3)(2007), 1231-1298.

[7] Moisil, Gr., Théodoresco, N., Functions holomorphes dans l'espace, Mathematica (Cluj), 5(1931), 142-159.

[8] Plaksa, S.A., Shpakovskii, V.S., Constructive description of monogenic functions in a harmonic algebra of the third rank, Ukr. Math. J., 62(8)(2011), 1251-1266.

[9] Schafer, R. D., An Introduction to Nonassociative Algebras, Academic Press, New-York, 1966.

[10] Schafer, R. D., On the algebras formed by the Cayley-Dickson process, Amer. J. Math., 76(1954), 435-446.

[11] Su Jin Lim, Kwang Ho Shon, Hyperholomorphic functions and hyperconjugate harmonic functions of octonion variables, J. Inequal. Appl. 2013:77.

[12] Xing-min Li, Zhao Kai, Li-zhong Peng, Characterization of octonionic analytic functions, Complex Variables, Theory and Appl., 50(13)(2005), 1031-1040.

\section{Cristina FLAUT}

Faculty of Mathematics and Computer Science, Ovidius University, Bd. Mamaia 124, 900527, CONSTANTA,

ROMANIA

http://cristinaflaut.wikispaces.com/

http://www.univ-ovidius.ro/math/

e-mail:

cflaut@univ-ovidius.ro 
cristina_flaut@yahoo.com

\section{Vitalii SHPAKIVSKYI}

Department of Complex Analysis and Potential Theory

Institute of Mathematics of the National Academy of Sciences of Ukraine,

3 , Tereshchenkivs'ka st.

01601 Kiev-4

UKRAINE

http://www.imath.kiev.ua/ complex/

e-mail: shpakivskyi@mail.ru 